Dawson, E; Jensen, E; (2011) Towards A Contextual Turn in Visitor Studies: Evaluating Visitor

Segmentation and Identity-Related Motivations. Visitor Studies, 14 (2) pp. 127-140.

10.1080/10645578.2011.608001. Downloaded from UCL Discovery:

http://discovery.ucl.ac.uk/1469309/.

\title{
ARTICLE
}

\section{Towards a 'contextual turn' in visitor studies: Evaluating visitor segmentation and identity-related motivations}

\author{
Emily Dawson ${ }^{1}$ and Eric Jensen ${ }^{2}$ \\ ${ }^{1}$ King's College London, London, UK. \\ ${ }^{2}$ University of Warwick, Coventry, UK.
}

\begin{abstract}
This paper assesses the use of audience segmentation in visitor studies by analyzing its application in the identity model of visitors proposed by Falk $(2007,2009)$. As a leading example of visitor segmentation, we examine this model's application in a specific case at US zoos to elaborate some of its limitations. Conventional short-term, episodic approaches to visitor research should be challenged and supplemented by a more contextually sensitive framework. We contend that segmentation approaches, and in particular Falk's theorization and operationalization of an identity model of visitors, are problematic. We argue for a 'contextual turn' that places visitors' experiences within a holistic and long-term framework of individual life circumstances, relationships and trajectories. Research and theory from education, sociology and cultural studies extends existing visitor research approaches by acknowledging complexity, change over time and the interwoven and developmental nature of socio-cultural variables influencing visitors' appropriation of new ideas and experiences.
\end{abstract}

Keywords: Audience segmentation, Identity, Visitor Studies, Museum, Zoo, Research Methods

\section{Towards a 'contextual turn' in visitor studies: Evaluating visitor segmentation and identity-related motivations}

Museum, gallery and zoo visitors have been the subject of a great deal of research interest in recent years. However, to date little headway has been made in terms of developing a rigorous and valid theoretical understanding of visitors to such cultural institutions, their reasons for visiting and the value such visits hold for them. In seeking to address these issues audience segmentation approaches, initially developed to model consumer behaviors for marketing purposes, have been adapted for visitor studies. Segmentation methods break visitors ${ }^{1}$ into sub-groups for purposes of analysis and intervention. While judicious use of taxonomic approaches can offer advantages for visitor studies and cultural institutions, we identify a number of disadvantages which undermine the value of audience segmentation for visitor research. In this paper we critique the theorization of audience segmentation currently being taken up by leading visitor studies researchers by exploring the identity model of museum visitors proposed by Falk (2009), as well as the development and application of this

\footnotetext{
${ }^{1}$ We appreciate that there are many kinds of public engagement institutions ranging from museums and art galleries, to science centres, zoos, aquaria, natural heritage site and festivals whose work is within the purview of visitor studies. In this paper we will refer to all such cases in terms of 'visiting' and 'cultural institutions' for the sake of simplicity.
} 
model in a specific case of visitor research conducted by Falk et al. (2007) at zoos in the United States.

At the outset, we wish to express our support of Falk's argument that the conventional shortterm, episodic approach to visitor research should be challenged and supplemented by a more contextually sensitive model. However, we contend that audience segmentation tools, and the specific way in which Falk has theorized and operationalized this shift are problematic and ultimately reductionist. In raising these points, we seek to promote a 'contextual turn' in visitor studies research, which abandons short-term, behaviorist approaches in favor of a model that authentically accounts for the ways in which museum visitors approach and make sense of their visits to cultural institutions and other sites for public engagement. To develop this argument, we examine Falk's model as a leading example of visitor segmentation approaches. In particular, we argue that the theorization of identity in Falk's model is not sufficiently pluralistic and developmental in nature, the rejection of demographic variables is premature, the claim to predictive power is counterfactual and the model's implementation in a major research project has been hampered by methodological and inferential errors that undermine Falk's claim that the model has been empirically validated. Recent book reviews have summarized Falk's identity model of visitors, highlighting the lack of evidence presented (Bickford, 2010) and the links between Falk's model and standard museum practice (McCray, 2010). We note Falk's acknowledgement of shortcomings within the model he proposed and we suggest that a more authentically contextual and systemic understanding of public engagement experiences within visitors' lives would overcome certain problematic elements of the model.

\section{Segmentation Approaches and Visitor Studies}

Originally a market research tool, audience segmentation techniques are not new to visitor studies. Rentschler (1998) suggested museums and similar institutions strategically adopted segmentation approaches as part of becoming commercially competitive organizations. This theme has been echoed by Horn (2006, p. 78) who positioned segmentation tools, and the motivational segmentation model developed for Museum Victoria, as a useful means of improving visitor experiences and developing "products and position in the leisure marketplace". Indeed, Black (2005, p. 11) went further and stated that, "No introduction to visitor studies can begin without a basic understanding of market segmentation."

Audience segmentation is believed to benefit commercial organizations seeking to improve their customers' experiences and their market share. Black $(2005$, p. 11) highlighted a number of characteristics that can be used to segment visitors, including demographic and geographic data, socio-economic, educational and hobby details and what he called a 'psychographic' approach, whereby visitors are grouped according to their "lifestyles, opinions, attitudes etc". It is this defining aspect of segmentation, where people are grouped according to specific characteristics (whether demographic or psychographic), where we see the disadvantages that are the focus of this article. In particular, we take issue with the decontextualization inherent in reducing people to their generic details, whether these are demographic characteristics or motivations. Such a process is essentialist, reductionist and ultimately of limited value to visitor researchers since it paints a misleading portrait of visitors and their experiences, while offering no portrait at all of those who do not visit.

To develop this critique, we turn to the application of segmentation methods to visitor studies within Falk's 'identity-related' visitor segmentation model. We focus on this model in particular because we believe the issues raised by Falk speak to a number of widely used concepts and tendencies within visitor studies. At the outset, we would like to express our support for the emphasis Falk places on the broader and longer term contexts of visits. While not novel in the broader field of educational research, this emphasis is worth adopting in a visitor research context, where much of the research is project specific and therefore narrowly framed in space and time (Economou, 2004; Jensen, 2011a). Despite the 
advantages offered by broadening the focus of visitor research, the identity segmentation model of visitors developed by Falk raises a number of theoretical and methodological questions. To what extent is his model of identity valid theoretically and methodologically? Is his side-stepping of standard demographic or contextual factors, such as race/ethnicity, gender, class and age justifiable? And finally, can visitors really be segmented into five identity-related groups, which predict their visiting patterns and outcomes?

We consider some of the underlying issues raised by Falk's model, while placing Falk's contribution into the broader context of educational (classroom studies), sociological and cultural research (Bennett et al., 2009; Bourdieu, 1984). In so doing, we are necessarily selective in our analysis of Falk's model, concentrating on a few issues we believe are particularly instructive for the development of a 'contextual model' that can guide visitor research.

\section{Audience segmentation: The identity model of museum visitors.}

The 'identity-related' visitor segmentation model proposed by Falk (2009) presents a welcome opportunity to discuss in more detail the use of segmentation as a research approach for visitor studies. Falk's model falls under Black's (2005) category of psychographic segmentations. Falk (2009, p.73) criticized the tendency of conventional museum visitor research to construct visitors in terms of their demographic characteristics such as age, gender, race/ethnicity, socio-economic status, social grouping and so on, or what he calls "big 'l' identities'". Instead, Falk segments visitors by their behavioral and selfreported characteristics, to identify and analyze what he describes as "little 'i' identities" ( $p$. 73). Falk posits five little ' $i$ ' identities which he has employed in his own research with museum visitors: the explorer, the facilitator, the experience seeker, the professional/hobbyist and the recharger (previously called a 'spiritual pilgrim', see Falk et al. 2007). This segmentation model is designed to take into account how visitors construct meaningful experiences before, during and after visits to cultural institutions.

Falk deploys the five 'identity-related' categories of museum visitors in his predictive model of visitor experiences, based on the relationships he outlines between the 'type' of visitor and their motivations for visiting a museum. He argues that museums would better serve their visitors by meeting the visit-based needs prompted by visitors' identity-related motivations. Falk (2009, p. 176) suggests that each visitor 'type' can be related to a typical visit trajectory for that 'type', that 'we can see that a visitor's entering identity-relative motivation predisposes the visitor to interact with the setting in predictable ways". Thus, it is argued that explorers tend to follow relatively similar 'explorer-trajectories' in their museum visits.

Falk proposes that while museum visits will always contain an unpredictable element, the available opportunities afforded by museums combined with an understanding of the five visitor types allow museums to predict most visitors' experiences and their longer-term outcomes. Therefore, although he notes certain problems with the model, Falk suggests this 'identity-related' segmentation model would enable museums to better anticipate the needs, trajectories and outcomes of their visitors' experiences, thus developing a provision that can be more tailored to the needs of different visitor types. This visitor segmentation model is in keeping with the use of such approaches in market research and commercial settings, wherein the audience 'segments' become the basis of tailored marketing and, to a lesser extent, service provision.

\section{Widening the analytical lens: the visitor in context}

Falk (2009) argued that the horizon of museum visitor studies should be broadened in two key directions: widening our understanding of the context of museum visits and concomitantly shifting how visitors are conceptualized. It is the argument, already widely accepted in other fields, for greater contextualization of museum visitor research that we see 
as the main positive contribution of Falk's model. Visitor research has traditionally tended to employ a short-term, positivist model of visitors that is located solely within the visit itself. This model assumes a priori the museum visit is a significant intervention in visitors' lives, and that this intervention will have an immediate, measurable effect that visitors can identify and self-report within the timescale of the visit itself. This model is operationalized through a number of methodological techniques based on pre- and post-visit testing of visitors' attitudes, knowledge, or both (Soren, 2007), often relying on self-report or psychometric scales. Building on his earlier work (e.g. Falk, 2004), Falk (2009) argues that extending the scope of visitor research to include a broader context provides a more valid way of investigating museum visiting.

For most people, museum-going is just a small slice of daily life, just one of many experiences in a lifetime filled with experiences...If we are to answer our fundamental questions of why people visit museums, what they do there, and what meaning they make of the experience, we must see the museum visitor experience as a series of nested, seemingly interrelated events. (Falk, 2009, pp. 34-35)

Thus Falk suggested museum visits should be viewed as related events in people's lives, rather than as stand-alone interventions with immediately identifiable impacts. Indeed, we would support his statement at the outset of the zoo study we examine below: "visitors do not arrive at a zoo or aquarium tabula rasa; they arrive with prior knowledge, experience, interest and motivations for their visit" (Falk et al., 2007, p. 6). Falk is not alone in promoting a more holistic view of such visits as embedded in complex and overlapping life contexts. However, his is the most recent and detailed application of this perspective to a model of visitors that has been widely circulated amidst visitor researchers and cultural institutions. By developing an awareness of how museum visits are integrated into visitors' lives over time, we agree with Falk that visitor research can develop far richer and more useful insights. However, we contend that such contextualization needs further development. For example, classroom educational research offers some initial ideas about how context might be better understood and applied to theorize the impact of visit experiences.

Education research has developed a broad, long-term perspective on people's experiences in particular learning-oriented environments, especially in the field of classroom studies. For example, Wortham's $(2000,2007)$ ethnographic classroom studies highlight the relevance of multiple timescales, including those of the local research site, students' homes and longer political timeframes, when exploring situated practices and how events are positioned and understood by those involved. Although Falk considered some contextual variables, for Falk the visits remain unquestionably the site of primary interest, still positioned broadly as an inherently significant learning intervention, and not as simply one event amongst many in a visitor's life. In contrast, when researching learning in science classrooms Lemke (2000, p. 284) argued that meanings are made and remade as "the trajectory of the developing social person takes him or her from classroom to classroom, from school to schoolyard, to street corner, to home, to the shopping mall, to TV worlds". Thus, although there is some acknowledgement of the broader context in which museum visits are situated for visitors, Falk's model is still limited by a primary focus on the institution rather than visitors' lives.

\section{Reconceptualizing visitors: Identity and difference in visitor research}

The second contribution of Falk's model we wish to highlight is his reconceptualization of museum visitors to acknowledge the relevance of visitor identities to visitor studies. One of the key arguments underpinning Falk's 'identity-related' visitor segmentation model is that personal identities of museum visitors can be related to their motivations for visiting, and when combined with the experiences offered by museums, can be used to understand and predict their visit trajectories and outcomes. While we question the conceptualization of 
those motivations and the claim to prediction, we endorse Falk's emphasis upon people's everyday behaviours, attitudes and needs.

The type of identity that does figure prominently into the myriad everyday decisions in our lives, including leisure, are what I have called "little 'i' identities" - identities that respond to the needs and realities of the specific moment and situation. (Falk, 2009, p. 73)

In this respect Falk has much in common with Hood (1993, p. 78), another museum visitor researcher whose work (drawn on by Falk) is informed by what she described as "psychosocial" characteristics based on her analysis of people's perceptions of themselves, their attitudes, personality and social behaviors. Both Falk and Hood have emphasized that museum visitors can be grouped according to their personalities and interests, rather than their demographic characteristics (Falk, 2009; Hood, 1993).

Falk cites and criticizes a tendency in museum visitor studies to categorize visitors in terms of demographic characteristics including gender, age, ethnicity and so on. Falk argues that demographics do not help museums understand their visitors: "it is not just race/ethnicity that provides a poor explanation for museum-going, so too do other demographic variables such as age, income, and education" (Falk, 2009, p. 29). In understanding museum visitors as individuals with personalities, attitudes and interests, Falk does visitor studies a great service by lending his support to challenging approaches that pigeon-hole visitors. However, it is in this part of Falk's model that further tensions begin to emerge, between the theories informing his model, and the model itself. Of these we unpick one issue around the averred identity-related motivations, concomitant problems with employing little 'i' categories, and issues raised by dismissing demographic factors.

In developing his model of five museum visitor identities, Falk cites a great deal of identityrelated research. Of these we concentrate on some of the ideas developed by Holland et al. (2001), which Falk (2009, p. 72) references in his discussion of the "view that identity is the combination of internal and external social forces - both cultural and individual agencies contribute to identity". What Holland et al. (2001) have highlighted in their work, drawing on sociology, psychology and cultural studies, is the complicated, nuanced and fluid nature of identity as a social product in practice. It is within this changing landscape of multiple selves and identity construction that tensions arise between the theoretical formulations of identity presented by Falk (2009, p.158) and the five categories he describes when he argues that "most identity-related museum motivations fall into one of five categories: Explorer Facilitator - Experience seeker - Professional/Hobbyist - Recharger". Given the theories Falk (2009) cites in his discussion of identity, it seems incongruous to conclude that museum visitors, by and large, can fit into five identity-related motivation boxes (albeit with some room for maneuver between boxes, and as Falk acknowledges but does not fully develop, the possibility to be simultaneously in multiple boxes). Thus, despite a discussion of the varied, changeable and contextual nature of identity construction, in his attempt to develop a model for practical use, Falk is ultimately too reductive in his treatment of the complexity of visitors' experiences and averred identity-related motivations.

A further tension within Falk's model can be located in the de-emphasizing of demographic factors' role in identity construction. Demographic factors such as gender, sexuality, age, ethnicity, ability/disability and socio-economic status have been described by Holland et al. (2001, p. 271) as "the second context of identity...positionality". Researchers have explored the relationship between demographic factors and behaviors, attitudes, expectations and experiences over a number of years and across a number of fields (e.g. Sandell, 2002). In his model, Falk (2009) explicitly dismisses demographic factors, which Holland et al. (2001, p. 271) described as being "the more durable social positions" and the role such factors have to play in understanding visitors. Although we would join Falk in questioning the use of 
demographic factors as the only basis for visitor segmentation, by dismissing these factors altogether Falk's model is self-limiting and incomplete.

It is highly problematic that Falk's (2009) model ignores the importance demographic factors hold for visitors and that it assumes visits to cultural institutions are perceived in similar ways regardless of ethnicity, age, class background or personal history. Demographic factors influence people's attitudes, experiences and behaviors, as demonstrated by a wealth of research in sociology, cultural studies and educational research, as well as in visitor research. For example, although criticized by Falk (2009, p. 37) as being concerned with "an esoteric philosophical analysis", Bourdieu's work on cultural habitus has demonstrated the relevance of social class as a crucial upstream factor in how people construct experiences of museums and other cultural offerings. In their large-scale study of European art museums, Bourdieu and Darbel (1991/1969) concluded that class was a paramount factor in both the enjoyment of art and in explaining non-visiting patterns (Jensen, 2010). They argued that the promise of "'museums for all' is in practice "false generosity, since free entry is also optional entry, reserved for those who, equipped with the ability to appropriate the works of art [through their middle or upper class upbringing], have the privilege of making use of this freedom" (p. 113). Moreover, Bourdieu's classic study Distinction (1984) in which he demonstrated the role of 'taste' in constructing cultural distinctions in favor of the upper classes was recently recreated in a contemporary UK context and the findings emphasized again the important effects of social class and people's attitudes and recreational activities (Bennett, et al., 2009).

Ethnicity is of critical importance in terms of how different aspects of culture are perceived and experienced (Hall, 1996, 1997). In educational contexts, research has indicated that ethnicity, gender and religious affiliation are influential factors in students' behaviors, selfperception, attainment and future choices (Archer, 2003; Corrigan, Dillon, \& Gunstone, 2007). Furthermore, in the context of visitor research the role of factors like ability/disability and ethnicity has been well established (Golding, 2009; Sandell, 2002).

While attending to 'psychographic' variables is useful, the attempt to dismiss these crucial sociological factors entirely from his analysis is problematic. Social justice theorists advocating the recognition of difference in culture and politics have argued that underestimating the importance of differences between people, whether in terms of their class, ethnicity, gender, sexuality or other factors, can create damagingly homogenous constructions, where those who do not fit in are simply ignored (Benhabib, 1996; Fraser \& Honneth, 2003; Young, 1990). Therefore, by purposely overlooking demographic factors Falk's identity model incurs limitations at the theoretical, methodological and political levels.

Related to problems associated with the dismissal of demographic variables is the failure of the model to account for non-visitors to museums and other cultural institutions. There is an unsurprisingly strong tendency in museum visitor research to focus on those who already visit cultural institutions. Unlike Hood (1995), who argued that museums ought to carry out research on communities instead of limiting themselves to their current visitors, Falk's theory focuses only on people who do visit museums.

As a result of the shortcomings identified above, we suggest that Falk's model begins with a flawed premise.

"The model postulates that virtually all people who visit museums begin from a relatively common, culturally-shared frame of reference museums are leisure educational institutions that afford a suite of possible benefits" (Falk, 2009, p. 173). 
We suggest that Falk has overestimated the degree to which there is a shared frame of reference across different groups, for example, across groups of varying socio-economic status or groups from minority ethnic backgrounds. This issue is particularly problematic because Falk (2009) applies his model to the topic of developing new audiences for cultural institutions. Falk's model, including the assumption of a culturally shared frame of reference, was devised based on research with existing museum visitors, yet he assumed that its validity holds for non-visitors as well.

Therefore, we suggest that the way in which Falk has theorized identity, with a focus on "little 'i'" segmentation at the expense of the "big 'l'", is problematic (p. 73, 2009). We see great promise in developing visitor studies beyond the limits of purely demographic analysis. However, we propose the more useful development would be a model combining demographic variables with other aspects of identity, rather than pursuing one at the expense of the other. We acknowledge that the complexity involved in Holland et al.'s (2001) model of identity may not be easy to apply within visitor studies. Concepts such as the 'figured world', 'positionality', the 'space of authoring' and the 'making of worlds' (Holland, et al., 2001, pp. 272-273) cannot be neatly diagrammed. Likewise, Holland et al.'s appreciation of improvisation and acknowledgement of change and fluidity does not result in a simple model of visitors to cultural institutions. Nevertheless, we contend that exploring how visitors and non-visitors understand cultural institutions is neither a neat nor simple undertaking, and to do justice to such complex processes requires a sufficiently complex view of identity.

\section{Operationalizing Identity-related motivations}

In order to demonstrate the limitations of the identity segmentation model of museum visitors it is important to consider the way in which these ideas have been put into practice. Falk (2009) provided limited evidence of the empirical research on which he has based his theoretical model (Bickford, 2010). Therefore the case we explore here is one of several studies that has employed Falk's identity-related segmentation model, a landmark zoo visitor study dubbed the multi-institutional research program (MIRP). In this multi-part study, Falk et al. (2007) set out to evaluate zoo visitors' motivations for attending and any changes in conservation-related attitudes or knowledge. Falk defined this task in terms of 'identityrelated motivations'. The focus on these motivations is explained in positivist terms as a prerequisite for 'prediction' of visitor outcomes: "we need to capture the essence of what motivates visitors so we could better predict what they might gain from their visit" (Falk, et al., 2007, p. 6).

In order to access the 'essence' of visitor motivation, the first phase of this three-year study comprised a "confirmatory study" to establish the validity and reliability of Falk's proposed 'five identity-related motivations' (Falk, et al., 2007, p. 15). This phase began with the selection of $100+$ statements of reasons or motivations for attending the zoo. Different subsets of these statements were then tested at different zoo and aquarium locations using statistical methods such as factor analysis to identify the items with the greatest internal reliability. Following these activities, Falk et al. (2007, p. 15) claimed to have identified a 20item scale comprising a "single measure for validly and reliably capturing zoo and aquarium visitors' identity-related motivations". Phase 2 of the research then used this 20 -item scale as an independent variable in concert with other scales intended to measure 'cognitive development' and 'affective response' by having respondents select and rate five items that most closely matched their reasons for attending that day.

\section{Essentialism in the identity-related segmentation model: Towards development}

Falk et al.'s (2007, p. 9) fundamental thesis was that visitors arrive at museums or zoos with "specific identity-related-motivations and these motivations directly impact how they conduct their visit and what meaning they make from the experience". He developed this thesis with his audience segmentation approach, described above, and referred to visitors as belonging 
to one of his five categories (or 'segments'). The problem of reductionism is evident in Falk et al.'s (2007, p. 15) study, which began its methodological discussion by reducing the initial aim of developing "a meaningful categorization of visitors based on their knowledge, interests, beliefs, attitudes, behaviors, and motivations" to an "instrument development" task based on Falk's previously developed 'five identity-related motivations' framework. At the outset, it is assumed that "many of these multiple 'entry' variables could be successfully subsumed into a single, multi-dimensional variable related to visitor's [sic] identity-related motivations" (Falk, et al., 2007, p. 15). In referring to the knowledge, interests, etcetera of visitors as 'entry' variables, Falk is recapitulating the kind of narrow vision of the visit from an institutional perspective rather than carrying out the kind of research that should begin with the lives of visitors and seek to understand the role of these institutions within this longer term 'whole life' framework.

In the MIRP study, Falk et al. claimed to have developed a valid and reliable instrument for capturing the identity-related motivations of zoo visitors. However, validity is not addressed by the instrumental form of psychometric scale development employed in this study. The methodological effort and evidence is focused on the internal reliability of the scale. It was not demonstrated, for example, through systematic qualitative pilot research, whether the selected statements used in the psychometric scale were understood as intended by visitors, nor was any inter-coder reliability reported to demonstrate that the categorization of different statements as one or another identity-related motivation was valid. That is, the validity of the instrument - or degree to which the selected statements measure what they are intended to measure (Jensen \& Holliman, 2009) - remains unproven in this research.

The operationalization of Falk's model is also undermined by inferential errors that exaggerate the quality of the evidence supporting this approach. Although Falk (2009) made claims about the predictive power and validity of his proposed identity-related motivations model, the empirical evidence within the MIRP study does not seem to support these claims. For example, at the outset of the MIRP study, Falk et al. stated "the motivations of the vast majority of visitors appeared to cluster around just a few identity-related motivations" (Falk, et al., 2007, p. 7). Despite the use of closed-ended methods wherein respondents had to choose five statements from within the 25 statements employed in Falk et al.'s scale, this clustering was not as widespread as averred. Namely, by Falk et al.'s (2007, p. 10) own unspecified standard, less than half $(48 \%)$ of their respondents "began their zoo or aquarium visit with a single, dominant identity-related motivation; the rest possessed multiple motivations for visiting". Falk does not satisfactorily explain this discrepancy nor fully integrate it in his theory.

This questionable interpretive approach can be seen in the authors' explanation for the finding that the two aquariums studied had different visitor motivation profiles: "these differences may have been due to the fact that data were collected in different seasons rather than representing a real difference in the profiles of aquarium visitors" (Falk, et al., 2007, p. 10). Here Falk introduces a new unaccounted for variable - the seasons - without any empirical elaboration, nor does he explicitly allow for it in the theory he goes on to develop. This empirical finding may undermine Falk's thesis that these motivations are in any way tied to identity. Yet, this disconfirming finding was not accepted as undermining Falk's thesis. Such handling of results is inconsistent with the very hypothetico-deductive methodological approach underpinning the study's emphasis on scale development over meaning and interpretation. Instead, it signals a commitment to the 'five types' model regardless of negative empirical findings.

Falk et al. (2007, p. 14) made other claims in this study that exceed their evidence (Marino, Lilienfeld, Malamud, Nobis, \& Brogliod, 2010). For example, it is claimed that the study "shows that individuals not only choose to visit or not visit zoos and aquariums based upon these identity-based motivations" (Falk, et al., 2007, p. 14). This quote makes a claim about 
the decision not to visit a zoo or aquarium, yet the study includes no data from non-visitors. That is, the research only accessed individuals already visiting a zoo or aquarium and therefore does not provide evidence about the non-visiting public. It is also claimed that "segmenting visitors by identity-related motivations (Explorers, Facilitators, et al.) provided the best way to understand both what visitors did in the institution as well as the short and long-term meaning they made from the experience" (Falk, et al., 2007, p. 10). However, this study does not report testing other variables (e.g. demographic) or attempt an amalgamation of demographic and motivational variables as we suggest in this article, thus raising unanswered questions about the basis of Falk et al.'s claim that the 'five types' approach is "best". Moreover, Falk et al. did not develop valid and convincing evidence of 'what visitors did in the institution' or of 'long-term meaning' (p. 10). As such, this headline conclusion from the MIRP study is questionable at best. Overall the MIRP example evinces the systematic use of problematic assumptions and inferences to validate the five identity-related motivations thesis, regardless of potentially contradictory evidence. When the theoretical and conceptual problems of this thesis are also taken into account, there is good reason to cast doubt on the five motivations and their averred link to identity, as well as claims made about their predictive power.

\section{Conclusion}

As we have argued above, Falk's identity-related visitor segmentation model contributes to challenging the mainstream visitor studies framework, which focuses narrowly on the duration of the visit. Such an episodic framework should be rejected, and Falk (2009) supports taking a broader perspective. Indeed, widening the analytical lens to include visitors' interests and motivations based on factors outside of the cultural institutions is likely to be much more fruitful in developing an accurate understanding of such institutions' impacts.

However, we identified a number of limitations in the theorization and application of Falk's visitor segmentation model. The small 'i' identities that are proposed to comprise these visitor segments are reductionist. Such segmentation essentializes and reifies visitors' identities, directing attention away from the diverse multiplicity of motivations, interests, desires and needs that draw individuals to engagement experiences in cultural institutions (e.g. Holliman, Colllins, Jensen, \& Taylor, 2009). In practice this approach minimizes the empirically demonstrated pattern of development in visitor expectations, perceptions and mindsets before, during and after visits to cultural institutions (Jensen, 2009; Jensen, 2010; Wagoner \& Jensen, 2010). As Falk (2009) himself ultimately acknowledges, individuals do not maintain a stable and unchanging orientation towards the visit. Rather visitors' initial expectations can change as they encounter new ideas and experiences (Wagoner \& Jensen, 2010).

Falk's visitor segmentation model summarizes large groups of people under the auspices of 'five types' which are then intended to provide the basis for museums' "interpretation, marketing, evaluation and even fund-raising" (Falk, et al., 2007, p. 14). The claim to have exhausted the range of possible visitor motivations with these types is particularly at odds with other studies that showed a much wider range of reasons for attendance at museums and other cultural institutions (Jensen, 2010; Jensen, 2011b; Packer, 2008; Packer \& Ballantyne, 2002; Yalowitz, 2002). The very concept that all visitors can be reduced to 'types' essentializes these individuals and downplays the richness and complexity of their life contexts. The result of this essentialism is an exclusionary framework that inaccurately demarcates the boundaries of possible visitor identities.

Falk's 'Personal Context' variables, or little 'i' characteristics, are said to have a predictive power that demographic variables do not: "Unlike demographic variables, Personal Context variables have the potential to predict changes in visitor knowledge and conservation attitudes" (Falk, et al., 2007, p. 6). However, the a priori rejection of demographic variables in 
favor of the 'five identity-related motivations' model entails a concomitant rejection of all the crucial insights about the complex role of class (e.g. Willis, 1977), educational attainment (e.g. Bourdieu \& Darbel, 1991/1969), social exclusion (e.g. Baumann, 1996; Jensen, 2010) and other socio-cultural factors in the decision to attend or not and the ultimate value of cultural institution visits for particular individuals. As Falk does not advocate collecting data on such variables there is a substantial risk that cultural institutions may fail to acknowledge weaknesses in, for example, reaching disadvantaged with low educational attainment.

We challenge Falk's dismissal of demographic variables and exclusive focus on current visitors. In contrast we advocate for a more inclusive approach that incorporates motivations, identities and sociological variables such as class and ethnicity. Such an inclusive approach would provide a much better basis for researching non-visitors as well. Falk's proposed five types (or 'segments') of museum visitor identity-related motivations is too restrictive and does not sufficiently take account of the development and change in motivations during and after the visit, thus contradicting Falk's theoretical emphasis on the need to broaden the context in which visitor research is carried out. Finally, given the many factors not accounted for in Falk's segmentation model, and a review of its development and use in practice in the MIRP, we suggest that the claim that it is predictive is not sustainable. Rather than seeking 'prediction' within a behaviorist market research framework, we propose that visits to cultural institutions should be understood within a holistic and long-term framework of individual life circumstances, relationships and trajectories.

Our proposed approach would acknowledge complexity, change over time and the interwoven and developmental nature of socio-cultural variables (Jensen \& Wagoner, 2010) influencing visitors' appropriation of new ideas and experiences encountered at a cultural institution (Wagoner \& Jensen, 2010). Such research would be inclusive, rather than exclusionary, and sensitive to difference as an important issue for cultural institutions to face. Such a 'contextual turn' in visitor studies could also inform the difficult task of widening access and participation amongst individuals and groups that have long been excluded from the sphere of cultural engagement (Friedman, 2007; Garibay, 2009; Gurian, 2006; Jensen, 2010; Lynch, 2001)

\section{References}

Archer, L. (2003). Race, Masculinity and Schooling. Maidenhead: Open University Press.

Baumann, G. (1996). Contesting culture: Discourses of identity in multi-ethnic London. Cambridge: Cambridge University Press.

Benhabib, S. (1996). Democracy and Difference. Princeton Princeton University Press.

Bennett, T., Savage, M., Silva, E., Warde, A., Gayo-Cal, M., \& Wright, D. (2009). Culture, class, distinction. Abingdon and New York: Routledge.

Bickford, A. (2010). Identity and the museum visitor experience. Curator: the Museum Journal, 53(2), 247-255.

Black, G. (2005). The engaging museum: an interpretive approach to visitor involvement. Abingdon and New York: Routledge.

Bourdieu, P. (1984). Distinction: A social critique of the judgement of taste (R. Nice, Trans.). London: Routledge and Kegan Paul Ltd.

Bourdieu, P., \& Darbel, A. (1991/1969). The love of art: European art museums and their public (C. Beattie \& N. Merriman, Trans.). Cambridge: Polity Press.

Corrigan, D., Dillon, J., \& Gunstone, R. (Eds.). (2007). The re-emergence of values in science education. Rotterdam: Sense Publishers.

Economou, M. (2004). Evaluation strategies in the cultural sector: The case of the Kelvingrove Museum and Art Gallery in Glasgow. Museum and Society, 2(1), 30-46.

Falk, J. (2004). The Director's Cut : Towards an Improved Understanding of Learning from Museums. Science Education, 88(1), 83-96.

Falk, J. (2009). Identity and the museum visitor experience. Walnut Creek: Left Coast Press. 
Falk, J., Reinhard, E., Vernon, C., Bronnenkant, K., Heimlich, J., \& Deans, N. (2007). Why zoos and aquariums matter: Assessing the impact of a visit to a zoo or aquarium. Silver Spring, MD: Association of Zoos \& Aquariums.

Fraser, N., \& Honneth, A. (2003). Redistribution or Recognition? London: Verso.

Friedman, A. J. (2007). The great sustainability challenge: how visitor Studies can save cultural institutions in teh 21sst Century. Visitor Studies, 10(1), 3-12.

Garibay, C. (2009). Latinos, Leisure values, and decisions: Implications for informal science learning and engagement. The Informal Learning Review, 94, 10-13.

Golding, V. (2009). Learning at the Museum Frontiers: Identity, Race and Power. Farnham: Ashgate.

Gurian, E. (2006). Civilizing the museum: the collected writings of Elaine Heumann Gurian. Abingdon and New York: Routledge.

Hall, S. (1996). New ethnicities. In D. Morley \& C. Kuan-Hsing (Eds.), Stuart Hall: Critical dialogues in cultural studies (pp. 441-449). London: Routledge.

Hall, S. (Ed.). (1997). Representation: cultural representations and signifying practices. London: Sage.

Holland, D., Skinner, D., Lachiotte Jr., W., \& Cain, C. (2001). Identity and agency in cultural worlds. Cambridge, MA: Harvard University Press.

Holliman, R., Colllins, T., Jensen, E., \& Taylor, P. (2009). ISOTOPE: Informing Science Outreach and Public Engagement. Final Report of the NESTA-funded project. Milton Keynes: The Open University.

Hood, M. (1993). After 70 years of audience research, what have we learned? Visitor Studies: Theory, Research and Practice, 15, 17-27.

Hood, M. (1995). A view from 'outside' research on community audiences. Visitor Studies: Theory, Research and Practice, 7, 77-87.

Horn, B. (2006). Barriers and drivers: building audience at the Immigration Museum, Melbourne, Australia. Museum International, 58(3), 78-84.

Jensen, E. (2010). Cambridge Science Festival: External evaluation report. Cambridge: University of Cambridge.

Jensen, E. (2010). Something Different: A pilot study evaluating family outreach activities at the Fitzwilliam Museum. Paper presented at the Fitzwilliam Museum, Cambridge.

Jensen, E. (2011a). Evaluate impact of communication. Nature, 469, 162.

Jensen, E. (2011b). Upstream Public Engagement at the Zoo: The Durrell Wildlife Park Public Participation Meeting. Jersey, Channel Islands: Durrell Wildlife Conservation Trust.

Jensen, E., \& Holliman, R. (2009). Investigating science communication to inform science outreach and public engagement. In R. Holliman, E. Whitelegg, E. Scanlon, S. Smidt \& J. Thomas (Eds.), Investigating science communication in the information age: Implications for public engagement and popular media (pp. 55-71). Oxford: Oxford University Press.

Lemke, J. L. (2000). Across the scales of time: Artifacts, activities, and meanings in ecosocial systems. Mind, Culture, and Activity, 7(4), 273-290.

Lynch, B. (2001). If the Museum is the Gateway, who is the Gatekeeper? Engage(11), 1221.

Marino, L., Lilienfeld, S. O., Malamud, R., Nobis, N., \& Brogliod, R. (2010). Do zoos and aquariums promote attitude change in visitors? A critical evaluation of the American Zoo and Aquarium study. Society \& Animals, 18, 126-138.

McCray, K. (2010). 'Identity and the Museum Visitor Experience'. Visitor Studies, 13(1), 121 124.

Packer, J. (2008). Beyond learning: Exploring visitors' perceptions of the value and benefits of museums experiences. Curator, 51(1), 33-54.

Packer, J., \& Ballantyne, R. (2002). Motivational factors and the visitor experience: A comparison of three sites. Curator, 45(3), 183-198.

Rentschler, R. (1998). Museum and performing arts marketing: A climate of change. The Journal of Arts Management, Law and Society, 28(1), 83-96. 
Sandell, R. (2002). Museums and the combating of social inequality: roles, responsibilities and resistance. In R. Sandell (Ed.), Museums, Society, Inequality (pp. 3-23). London: Routledge.

Soren, B. (2007). Audience-based measures of success: Evaluating museum learning. In B. Lord (Ed.), The manual of museum learning (pp. 221-252). Plymouth: Altamira Press.

Wagoner, B., \& Jensen, E. (2010). Science learning at the zoo: Evaluating children's developing understanding of animals and their habitats. Psychology \& Society, 3(1), 65-76.

Willis, P. (1977). Learning to labour. How working class kids get working class jobs. Farnborough, Hants: Saxon House.

Wortham, S. (2000). Interactional positioning and narrative self-construction. Narrative Inquiry, 10(1), 157-184.

Wortham, S. (2007). Shifting identities in the classroom. In A. Caldas-Coulthard \& R. ledema (Eds.), Identity trouble: Critical discourses and contested identities (pp. 205-228). New York: Palgrave Macmillan.

Yalowitz, S. (2002). Personality and motivation in visitor satisfaction. Visitor Studies Today, 2002, 14-17.

Young, I. (1990). Justice and the Politics of Difference. Princeton: Princeton University Press.

\section{About the authors}

Emily Dawson is completing a PhD at King's College London: emily.dawson@kcl.ac.uk.

Dr. Eric Jensen (http://warwick.academia.edu/EricJensen) is Assistant Professor of Sociology at the University of Warwick, where he teaches doctoral and master's level social research methods and co-convenes the Master of Science program in Science, Media \& Public Policy: e.jensen@warwick.ac.uk. 